\title{
Herramientas de Gestión Ambiental Para las Carreteras de Cuarta Generación (4G) en Colombia
}

\section{Environmental Management Tools for Roads Fourth GenERATION 4G IN COLOMBIA}

\section{'William Eduardo Mosquera Laverde, ${ }^{2}$ Oscar Alejandro Vásquez Bernal}

\author{
${ }^{1}$ Universidad Cooperativa de Colombia, Facultad de Ciencias \\ Administrativas, Bogotá D.C., Colombia. \\ ${ }^{2}$ Universidad Nacional Abierta y a Distancia-UNAD, Escuela de Ciencias Básicas \\ Tecnologías e Ingenierias, Bogotá D.C., Colombia \\ ${ }^{1}$ williame.mosquera@campusucc.edu.co \\ ${ }^{2}$ oscar.vasquez@unad.edu.co
}

\section{RESUMEN}

En este artículo se pretende analizar la aplicación del Manual para la asignación de compensaciones por pérdida de biodiversidad, en el sector de infraestructura vial en Colombia. Para ello, se realizó una revisión bibliográfica sobre el estado del arte acerca de la biodiversidad, servicios eco sistémicos (SE), pérdidas de biodiversidad, las compensaciones por pérdida de biodiversidad y los impactos ambientales ocasionados por las vías, con el fin de analizar los principios de compensación definidos por la normatividad colombiana a la luz de las transformaciones del paisaje ocasionadas por la fragmentación de ecosistemas. Lo anterior, teniendo en cuenta, que durante la última década, la fragmentación se ha convertido en un problema ambiental global. Con base en lo anterior, la metodología empleada incluye una revisión y análisis del estado actual del arte a la luz de las compensaciones en el sector de infraestructura vial. Luego, se planteó una propuesta de gestión para la infraestructura vial en Colombia, que permita incluir la fragmentación de ecosistemas dentro del análisis de compensación para proyectos lineales de infraestructura vial, y, además, contribuir con la no pérdida neta de biodiversidad.

Palabras clave: biodiversidad, compensación, servicios ecosistémicos, infraestructura vial, no pérdida neta de biodiversidad. 


\section{Abstract}

It aims to analyze the application manual for the allocation of compensation for loss of biodiversity applied in the road infrastructure sector in Colombia. To do a literature review on the state of the art on biodiversity, ecosystem services (ES), loss of biodiversity, compensation for loss of biodiversity and environmental impacts caused by the way, in order to analyze the principles developed compensation defined by the Colombian regulations in light of the changes in the landscape caused by the fragmentation of ecosystems, considering that over the past decade, fragmentation has become a global environmental problem. Based on the above, the methodology includes a review and analysis of the current state of the art in light of compensation in the road infrastructure sector. Then a management proposal for the road infrastructure in Colombia, allowing include the fragmentation of ecosystems in the analysis of linear compensation for road infrastructure projects and contribute no net loss of biodiversity arises.

Keywords: biodiversity, compensation, ecosystem services, road infrastructure, no net loss of biodiversity.

\section{INTRODUCCIÓN}

En el ámbito mundial, la biodiversidad [1,2] ha sido reconocida no solo como expresión de las diferentes formas de vida presentes en la tierra, sino también como la base del bienestar y la calidad de vida de los seres humanos [3]. Este último aspecto, se define también como servicios eco sistémicos, indispensables para la supervivencia de la vida humana en el planeta, "algo sólo posible si se garantiza la estructura y el funcionamiento de la biodiversidad" [4].

Tal como se plantea en [4], existen cinco causas globales de pérdida de biodiversidad: pérdida y transformación ecosistemica, introducción de especies, sobreexplotación, contaminación y cambio climático. De estos motores de transformación, la pérdida y cambios de ecosistemas a través de la fragmentación de los mismos, se considera de origen antrópico y uno de los factores que genera alto impacto ambiental, razón por la cual, la inclusión de este factor en las estimaciones de compensación por pérdida de biodiversidad, podría aumentar el soporte en la toma de decisiones de las autoridades ambientales, así como lograr una aproximación más real a los impactos ocasionados.
Adicionalmente, la escala de la información para la toma de decisiones en el sector ambiental en Colombia, es muy baja (Mapa de ecosistemas continentales, costeros y marinos de Colombia, bajo un mismo sistema de coordenadas cartográficas, a escala 1:500.000) [5], lo que ha permitido que las afectaciones locales a la biodiversidad no sean fácilmente percibidas en el país. De acuerdo con el mapa, Colombia cuenta con 311 tipos de ecosistemas continentales y costeros, incluidas áreas naturales con poca transformación y paisajes transformados por actividades humanas de asentamiento, producción y extracción [6]; así mismo, teniendo en cuenta el área mínima cartografiable de 2.500 ha, los ecosistemas de menor tamaño no son representados ni tenidos en cuenta.

Es así, como en un intento para compensar la pérdida de biodiversidad neta en el país, el MADS, en compañía de The Nature Conservancy - TNC, World Wildlife Fund - WWF y Conservación Internacional - Cl, bajo la ejecución de TNC, desarrolló el Manual para la asignación de compensaciones por pérdida de biodiversidad, con el fin de determinar y cuantificar las medidas de compensación por pérdida de biodiversidad, 
respondiendo tres inquietudes fundamentales: cuánto compensar, dónde compensar y cómo compensar [7], bajo tres principios: jerarquía de mitigación, no pérdida neta de biodiversidad y equivalencia ecosistémica [8].

Este manual lo deben aplicar los solicitantes de licencia ambiental de competencia de la Autoridad Nacional de Licencias Ambientales - ANLA [9] y se incluye en la evaluación integral en el proceso de licenciamiento. De la misma manera, se constituye en una buena aproximación para la compensación en términos de área y representatividad de ecosistema (a escala de paisaje); sin embargo, aún tiene muchos vacíos en cuanto a cómo compensar la funcionalidad y la no pérdida de biodiversidad a una escala local.

Teniendo en cuenta lo anterior, en el presente trabajo se analizó la literatura existente sobre el impacto de la fragmentación de ecosistemas por proyectos de infraestructura vial [10], a la luz de los principios definidos por la normatividad colombiana para la estimación de las compensaciones por pérdida de biodiversidad, tratando de responder, por una parte, si son suficientes para la conservación de la biodiversidad, los enfoques actuales del Manual de compensación, aplicados a proyectos de infraestructura vial y, por otra, si deberían existir herramientas adicionales de gestión para contribuir a la no pérdida neta de biodiversidad, diferentes a la equivalencia ecosistémica planteada en el Manual.

Este documento es una aproximación inicial a la discusión sobre temas que aún no se han incluido dentro de la gestión de la biodiversidad en cuanto a lo normativo en Colombia. Así mismo, pretende plantear un debate sobre la importancia de contemplar factores adicionales, como la fragmentación, al momento de evaluar el impacto que se ha de compensar por proyectos de infraestructura vial y así contribuir a una no pérdida de biodiversidad a escala local. A partir de este análisis se propone un enfoque adicional para la gestión de la biodiversidad, incluyendo la fragmentación dentro de los parámetros de evaluación de compensaciones, de tal manera, que los esfuerzos para compensar este tipo de proyectos, se enfoquen en acciones de conservación in situ [1] con el fin de disminuir el impacto ocasionado por la fragmentación.

\section{Metodología}

\section{A. Tipo de estudio}

Para el presente trabajo se seleccionó la profundización temática [11], por lo cual se realizó una revisión bibliográfica del estado del arte, con el ánimo de plantear la discusión sobre si son suficientes para la conservación de la biodiversidad, los enfoques actuales del Manual de compensación, aplicados a proyectos de infraestructura vial y si deberían existir herramientas adicionales de gestión para contribuir a la no pérdida neta de biodiversidad, diferentes a la equivalencia ecosistémica planteada en el Manual. A continuación se describen los pasos que se siguieron durante el desarrollo del presente trabajo (Figura 1).

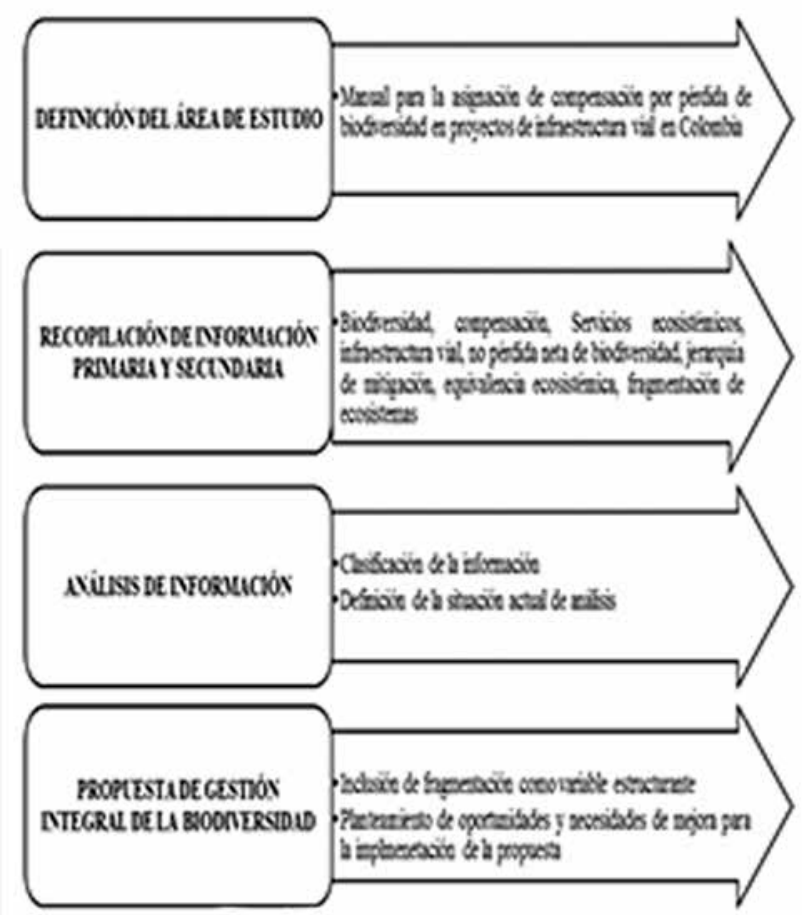

Fig. 1 Esquema metodológico 


\section{B. Definición del área de estudio}

Se realizó una revisión sobre el estado de aplicación del Manual para la asignación de compensación por pérdida de biodiversidad en proyectos de infraestructura vial en Colombia, con el fin de explorar si los enfoques actuales del Manual pueden llegar a alcanzar los impactos esperados en la no pérdida neta de biodiversidad en proyectos viales, encontrando que el tema de la fragmentación de ecosistemas aún no ha sido incluido dentro de las evaluaciones.

\section{Recopilación de información primaria y secundaria}

Luego se recopiló literatura existente sobre el tema, en la que se encontraron fuentes de información del Estado colombiano y documentos financiados por diferentes stakeholders, en relación con la infraestructura vial, para determinar cómo diseñar la gestión ambiental para proyectos viales. Con base en esto, se elaboró una propuesta inicial, que se plasmó en el marco conceptual sobre Biodiversidad y servicios ecosistémicos.

\section{Análisis de la información}

Se realizó la revisión de la información recolectada de diferentes fuentes con el fin de responder las preguntas de gestión planteadas en el presente documento. La información se clasificó en diferentes categorías: biodiversidad y servicios eco sistémicos, fragmentación, gestión integral de la diversidad biológica, infraestructura vial, sistemas de gestión ambiental y desarrollos viales actuales. Esta información se analizó y presentó en la sección de análisis de la situación.

\section{E. Propuesta de gestión integral de la biodi- versidad en infraestructura vial}

Con base en la información analizada, se realizó una propuesta de gestión integral de la biodiversidad para proyectos de infraestructura vial, en la que se incluye la fragmentación como variable estructurante para la adaptación de la biodiversidad basada en ecosistemas, frente a los megaproyectos viales por realizarse en el país en los próximos años.
Por último, se plantearon las oportunidades y necesidades de mejora para la implementación de la propuesta, como un aporte a las evaluaciones que se han de realizar en el marco del Manual para la asignación de compensación por pérdida de biodiversidad en relación con el desarrollo de proyectos viales, y de la misma manera, para contribuir con los objetivos de Colombia frente a la no pérdida neta de diversidad biológica.

\section{Resultados}

Como resultado del análisis de la información sobre el estado del arte de la compensación por pérdida de biodiversidad en los proyectos de información vial, se obtuvieron los siguientes planteamientos, que dinamizan la discusión sobre si son suficientes para la conservación de la biodiversidad, los enfoques actuales del Manual de compensación, aplicados a proyectos de infraestructura vial y si deberían existir herramientas adicionales de gestión para contribuir a la no pérdida neta de biodiversidad diferentes a la equivalencia ecosistémica planteada en el mismo.

\section{A. Estado del arte de la información}

1) Escala de análisis para la toma de decisiones. La información cartográfica utilizada para la toma de decisiones en cuanto a Ecosistemas en el ámbito nacional es a escala 1:500.000 [12], en la que se incluyen criterios de bioma, tipo de sistema ecológico y cobertura. Es aquí, cuando el reto para posteriores mapas de ecosistemas, además de desarrollar una escala más detallada, requiere la integración de leyendas más comprensivas que permitan integrar no solo patrones espaciales de los mismos, sino información sobre la composición y funcionamiento de los ecosistemas [10].

Información con mayor detalle, que podría ser incluida dentro de las estimaciones de las compensaciones, se encuentra en el mapa de cobertura y uso de la tierra, con base en la metodología 
Corin Land Cover para los periodos 2000 - 2002 y 2005 - 2009, a escala 1:100.000 [12].

2) Manual para la asignación de compensaciones por pérdida de biodiversidad en Colombia. El manual de compensación es un instrumento normativo cuyo objetivo es "establecer el procedimiento para la determinación y cuantificación de las medidas de compensación por pérdida de biodiversidad, y es un instrumento de uso obligatorio para los usuarios, en la elaboración de los estudios de impacto ambiental, para la Autoridad Nacional de Licencias Ambientales - ANLA, en la evaluación y aprobación de las medidas de compensación por pérdida de biodiversidad en el proceso de licenciamiento ambiental y sus modificaciones" [8]. Las medidas de compensación establecidas solo determinan la conservación efectiva en áreas ecológicamente equivalentes en donde se pueda conservar de forma permanente, según los criterios de cuánto, dónde y cómo.

El cálculo de las áreas por compensar se determina por medio de factores de compensación y tiene en cuenta los siguientes elementos: representatividad del ecosistema, rareza, remanencia y tasa de transformación anual, con una variación del factor total de compensación entre 2 a 10, de 2 a 5 para áreas con vegetación secundaria y de 4 a 10 para áreas con ecosistemas naturales [8].

Las compensaciones se desarrollarán en lugares dentro del portafolio de áreas prioritarias para la conservación, en la zona de influencia del proyecto afectante, cumpliendo los criterios de selección establecidos del área por compensar, como son: el mismo tipo de ecosistema, tamaño igual o mayor, contexto paisajístico, riqueza de especies e influencia del proyecto; de lo contrario, se desarrollará en zonas subhídricas cercanas o con influencia del municipio donde se lleve a cabo el proyecto, con actividades de restauración o manejo del paisaje y, por último caso, en las actuales áreas protegidas del SINAP [8].
Las acciones que se deben desarrollar para la compensación se encuentran descritas en la Figura 2.

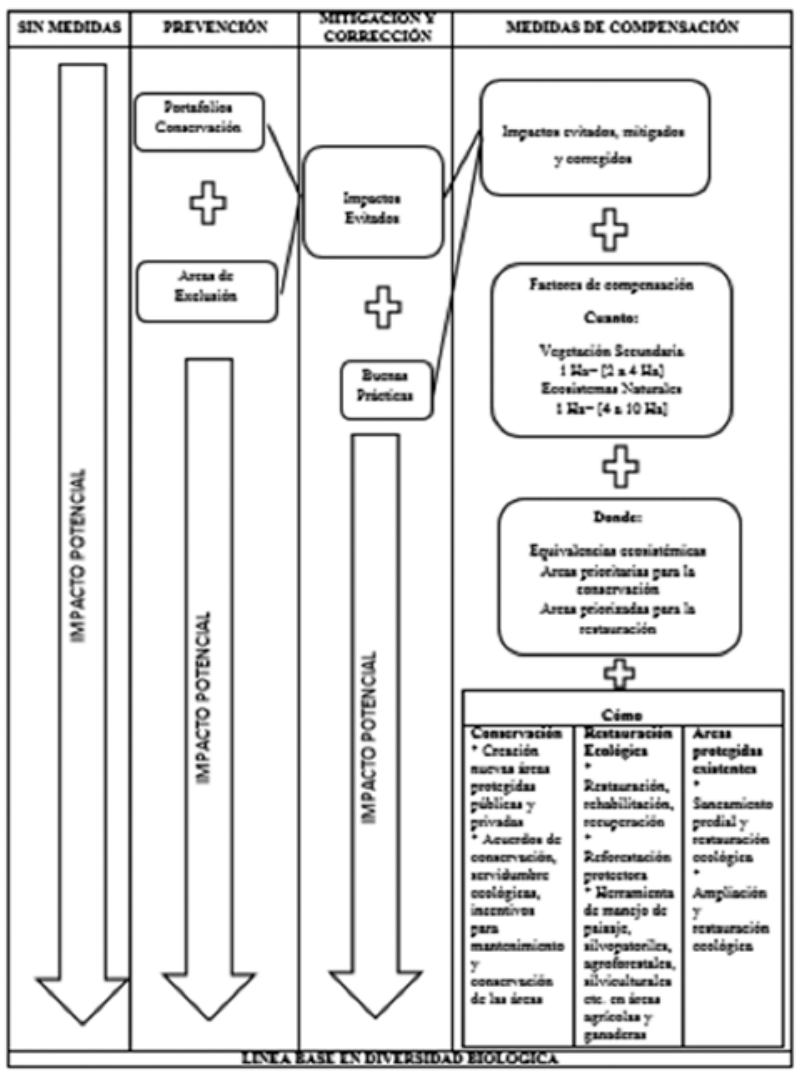

Fig. 2 Medidas de prevención, mitigación, corrección y compensación en proyectos, obras o actividades. Tomado de [8]

3) Gestión ambiental en la infraestructura vial. El eje central del Plan Nacional de Desarrollo Prosperidad para todos, es el crecimiento de la economía, mediante emprendimientos de extracción de los recursos naturales, construcción de infraestructura, agroindustria e innovación en ciencia y tecnología [10], razón por la cual, la infraestructura vial se convierte en un eje de desarrollo socio económico para los colombianos, que se encuentra en crecimiento.

Teniendo en cuenta lo anterior, y los impactos ocasionados en la biodiversidad por este tipo de proyectos, Invias y el Ministerio de Ambiente, Vivienda y Desarrollo Territorial [13], desarrollaron 
la Guía ambiental para proyectos de infraestrutura en el subsector vial. En este documento, se encuentra un capítulo sobre las medidas de manejo que deben ser tenidas en cuenta en las empresas que trabajan en el sector, las cuales se describen en la Tabla 1.

\section{TABLA I.}

Impactos y Medidas de Gestión del Subsector Vial en Colombia, Asociados a la Pérdida de Biodiversidad.

\begin{tabular}{|c|c|c|}
\hline \multicolumn{3}{|c|}{$\begin{array}{l}\text { IMPACTOS AMBIENTALES DE LA } \\
\text { INFRAESTRUCTURA VIAL }\end{array}$} \\
\hline $\begin{array}{l}\text { ELEMENTO } \\
\text { AMBIENTAL }\end{array}$ & $\begin{array}{l}\text { IMPACTO } \\
\text { AMBIENTAL }\end{array}$ & $\begin{array}{l}\text { DESCRIPCION DEL } \\
\text { IMPACTO }\end{array}$ \\
\hline \multirow{5}{*}{ BIÓTICO } & $\begin{array}{l}\text { A f e c t a ción } \\
\text { áreas ambien- } \\
\text { talmente sen- } \\
\text { sibles. }\end{array}$ & $\begin{array}{l}\text { Referido a cambios } \\
0 \text { afectaciones de } \\
\text { áreas definidas por } \\
\text { la ley como de im- } \\
\text { portancia ambiental } \\
\text { ó que cumplen una } \\
\text { función ambiental. }\end{array}$ \\
\hline & $\begin{array}{l}\text { Cambios en la } \\
\text { cobertura ve- } \\
\text { getal. }\end{array}$ & $\begin{array}{l}\text { Modificación en } \\
\text { áreas, de los di- } \\
\text { ferentes tipos de } \\
\text { vegetación. }\end{array}$ \\
\hline & $\begin{array}{l}\text { Alteración de } \\
\text { hábitat. }\end{array}$ & $\begin{array}{l}\text { Se refiere al daño } \\
0 \text { perturbación al } \\
\text { hábitat natural de } \\
\text { la fauna presente. }\end{array}$ \\
\hline & $\begin{array}{l}\text { D e s p I a z a - } \\
\text { miento de po- } \\
\text { blaciones fau- } \\
\text { nísticas. }\end{array}$ & $\begin{array}{l}\text { Se refiere al des- } \\
\text { plazamiento for- } \\
\text { zado de algunas } \\
\text { especies propias de } \\
\text { una zona cuando su } \\
\text { hábitat es alterado. }\end{array}$ \\
\hline & $\begin{array}{l}\text { Incremento de } \\
\text { demanda de } \\
\text { recursos natu- } \\
\text { rales. }\end{array}$ & $\begin{array}{l}\text { Necesidad del con- } \\
\text { sumo de recursos } \\
\text { naturales para la } \\
\text { ejecución del pro- } \\
\text { yecto alterado. }\end{array}$ \\
\hline PAISAJE & $\begin{array}{l}\text { Alteración de } \\
\text { los valores es- } \\
\text { cénicos de una } \\
\text { unidad de pai- } \\
\text { saje. }\end{array}$ & $\begin{array}{l}\text { Cambios en la visi- } \\
\text { bilidad, calidad vi- } \\
\text { sual e intervención } \\
\text { antrópica que afectan } \\
\text { el valor escénico. }\end{array}$ \\
\hline
\end{tabular}

Adicionalmente, para el presente estudio, vale la pena mencionar que en Colombia se emplea la figura concesión vial, como mecanismo para entregar por un tiempo determinado al sector privado una vía para su construcción, rehabilitación o mantenimiento. Esta consiste en una nueva forma de contratación del Estado con el sector privado, en la que, para el caso, el Instituto Nacional de Concesiones - INCO - paga la construcción de una carretera no en el mismo tiempo en el que esta se realiza (que sería la forma tradicional) sino en un plazo más amplio (15 o 20 años) dándole al concesionario (sector privado), como garantía de pago, lo recaudado por concepto de peaje en este tiempo. Es de aclarar que en este periodo el concesionario será el encargado del mantenimiento de la vía con los mismos recursos de peaje. Al final del tiempo, el proyecto revierte a la nación [14].

Según [14], el sistema de concesión está organizado en tres etapas de la siguiente forma:

Primera etapa. Pre construcción: diseño y programación. En esta se deben elaborar los estudios finales de impacto ambiental del proyecto.

Segunda etapa. Construcción: se deben implementar los programas establecidos en el diseño.

K Operación. Mantener, evaluar y monitorear los programas ambientales establecidos.

Este tipo de proyectos adjudicados por la modalidad de concesión, tienen asegurados los recursos económicos para su ejecución y, por ende, se espera sean los que mejor gestión ambiental puedan tener [14].

4) Fragmentación de ecosistemas - variable estructurante para compensaciones en proyectos viales. Por otro lado, el presente estudio 
TABLA II.

permitió identificar una variable estructurante que no ha sido incluida dentro del análisis de compensaciones por pérdida de biodiversidad, como lo es la fragmentación ocasionada por el paso de la vía, lo cual acelera cambios en los ecosistemas $\mathrm{y}$, por consiguiente, aumenta su vulnerabilidad a crisis ambientales [10]. Las obras de infraestructura generan fragmentación de los ecosistemas, reduciendo el hábitat original de las especies [15].

Adicional a esto, los estudios para evaluar los efectos de estas obras sobre la biodiversidad no son suficientes, y no hay monitoreo de los efectos en la biodiversidad después de la realización de las obras.

De acuerdo con [10], desde la década de los ochenta se viene investigando la relación entre el tamaño de los fragmentos de los bosques con la pérdida de especies, y se conocen algunos umbrales espaciales más allá de los cuales se produce el colapso de la biodiversidad. En este sentido, es importante el umbral temporal. Si bien muchos fragmentos podrían estar más allá del umbral especial, podrían no haber perdido todavía las especies y estar más acá del umbral temporal. Esto causa una gran incertidumbre para la gestión de la biodiversidad en escenarios que tienen el potencial de generar más fragmentación, como la expansión de forestería, la agricultura, la minería, la urbanización y el desarrollo hidroenergético y la construcción de infraestructura vial.

Adicionalmente al tamaño de los fragmentos, hay variables adicionales que permiten cuantificar los patrones del paisaje transformado y que se describen en la Tabla 2.

\section{Variables para cuanti- ficar los patrones del paisaje fragmentado}

Área de los parches (tamaño).

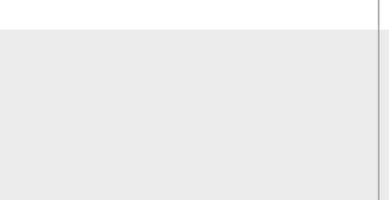

Efecto borde y área de hábitat interior (cantidad de área del parche que no sufre el efecto borde).

Distancia a parches de bosque y continuidad.

Medida de la fragmentación de un ecosistema (número de fragmentos).
Forma de los parches.

Variables por Tener en Cuenta Cuando se Evalúan Paisajes Transformados. [16], [17].

\section{Relevancia}

Factor importante cuando se consideran las probabilidades de conservar Spp típicos de ecosistemas maduros en paisajes de múltiples usos.

Los parches de ecosistemas presentan formas irregulares dejando percibir el patrón histórico de la fragmentación y la agresividad de los usos del suelo adyacente. Formas compactas son efectivas para proteger los recursos interiores contra efectos deleterios del ambiente exterior.

Se genera diferencias en la composición y abundancia del SPP en el borde debido a un aumento en la penetración de la luz y vientos, generando cambios de microclimas.

El valor de conservación de un determinado fragmento de bosque puede ser aumentado 0 degradado de acuerdo con el patrón de los otros elementos del paisaje a su alrededor. Las relaciones espaciales entre parches que restringen 0 facilitan el movimiento de los organismos en el paisaje determinan la persistencia regional de las poblaciones.

Indicación simple de qué tan fragmentado se encuentra un ecosistema en un área de estudio. A mayor número de parches, mayor fragmentación del paisaje. 
Las vías también han sido señaladas como causa directa de la deforestación [18], debido a que cambian la posibilidad de acceso de la población a los bosques. Los actuales planes viales que atraviesan zonas selváticas se constituyen en motores principales de degradación y pérdida de biodiversidad [19].

La vías provocan dos tipos de efectos de fragmentación, como son: el efecto barrera que se produce cuando se impide la movilidad de los organismos o de sus estructuras reproductivas, lo que trae como consecuencia limitar el potencial de los organismos para su dispersión y colonización. Las vías rompen la continuidad del dosel (estrato superior de los bosques) e interrumpen las posibilidades de movilidad de los animales. Las barreras también pueden restringir la habilidad de los organismos de encontrar sus parejas, lo que puede llevar a la pérdida de su potencial reproductivo.

El otro efecto es el de borde se presenta cuando un ecosistema es fragmentado y se cambian las condiciones bióticas y abióticas de los fragmentos y de la matriz circundante [20]. En el caso de las vías, este efecto se presentará en las inmediaciones o borde de la vía, en las que se crearán condiciones con mayor temperatura, menor humedad, mayor radiación y mayor susceptibilidad al viento. Este efecto de borde puede penetrar 50 $\mathrm{m}$ para aves, $100 \mathrm{~m}$ para los efectos micro climáticos y $300 \mathrm{~m}$ para insectos. Como consecuencia del mismo, se modifica la distribución y abundancia de las especies, cambiando la estructura de la vegetación $y$, por tanto, la oferta de alimento para la fauna.

Cabe anotar que los efectos anteriores también provocan en las especies atropellamientos y cambios en los patrones reproductivos ocasionados por el ruido, movimiento, vibraciones, las luces artificiales y la presencia humana.

Teniendo en cuenta que no se sabe a ciencia cierta cómo impactan los proyectos de desarrollo en términos de pérdida de biodiversidad y que actualmente se juega con una alta incertidumbre (escala de la información poco detallada), el Manual para asignación de compensación por pérdida de la biodiversidad, actualmente empleado en Colombia, es una buena herramienta para que las personas naturales o jurídicas que desarrollan proyectos que requieren licenciamiento ambiental, luego de aplicar la jerarquía de la mitigación, puedan realizar acciones encaminadas a la no pérdida neta de biodiversidad, a través de la equivalencia ecosistémica.

Para el caso de proyectos lineales, como lo son las vías, se han identificado algunas debilidades en la aplicación del Manual de compensación, tales como: las intervenciones se presentan en ecosistemas variados a través de la vía y en pequeños fragmentos (solo se incluyen dentro de los factores de compensación las áreas impactadas por la construcción de la vía e infraestructura anexa); la duración de las obras es corta (para lo cual el manual plantea que la compensación se realiza por el tiempo de vida del proyecto y no incluye la fase de operación para el caso de las vías) y no se tiene claridad sobre qué va a pasar después con las compensaciones, debido al corto tiempo empleado para compensaciones en infraestructura vial. Por otra parte, para el caso de las vías, no se contempla el impacto directo y adicional como consecuencia de la fragmentación de los ecosistemas.

Sumado a esto, las definiciones de las compensaciones usan criterios remotos (mapa de ecosistemas) y se estiman tomando como línea base la combinación de la cobertura y el tipo de bioma, sin tener una aproximación real y local del estado de los ecosistemas.

Es así, que para enriquecer esta discusión, se toma lo planteado por [21], quien afirma que "la biodiversidad es un producto único de la historia y un atributo singular de la geografía. La biodiversidad hoy es diferente de lo que fue en el pasado y también entre un lugar y otro. No 
existen réplicas para la biodiversidad en el espacio y tiempo. Cada lugar y tiempo es único en su diversidad particular".

Teniendo en cuenta lo antes mencionado, se requiere de herramientas complementarias al Manual de compensación que contribuyan a una gestión integral de la biodiversidad y que reduzcan los impactos reales de proyectos lineales como la infraestructura vial, en los que los impactos van más allá del área destinada a la construcción de la vía o a la infraestructura complementaria (campamentos, zonas de almacenamiento de materiales, entre otros), generando fragmentación de ecosistemas que en últimas se ve reflejado en una pérdida neta de biodiversidad.

Es así, como luego de realizar el análisis de la información, se propone que se incluya dentro de las variables estructurantes para las evaluaciones de compensación de biodiversidad, la fragmentación de ecosistemas, con el fin de contar con una evaluación integral y cercana a un impacto real de los proyectos de infraestructura vial. Los impactos en la fragmentación de ecosistemas naturales y seminaturales, deben ser más fuertes y contar con una temporalidad mayor, que los generados de manera directa por la construcción de las calzadas.

Adicionalmente, los impactos ocasionados por la fragmentación de los hábitats naturales se pueden mitigar mediante el diseño de redes ecológicas, las cuales se configuran como instrumentos útiles en el planeamiento territorial en áreas fragmentadas y contribuyen a la conservación in situ. Para ello, es necesario contemplar que el desplazamiento de las especies desde un fragmento forestal a otro a través de la matriz, depende de la distancia real existente entre los fragmentos, pero también de la percepción que de esta distancia tiene la especie en cuestión [22].

\section{Discusión de Resultados}

Teniendo en cuenta lo antes mencionado, la propuesta de compensación para proyectos de infraestructura vial, está plasmada en tres enfoques:

- Las medidas de compensación deben realizarse in situ, a través de la construcción de infraestructura que promueva la conectividad entre los parches generados por el paso de la vía, lo que reduciría realmente la no pérdida neta de biodiversidad, como lo plantea el manual.

- Las medidas de compensación deben realizarse de manera simultánea con la ejecución del proyecto (por ejemplo pasos subterráneos y aéreos para fauna) y entrar a ser parte de la infraestructura vial.

- Teniendo en cuenta que la infraestructurapara conservación de la biodiversidad va a ser parte de la infraestructura vial, se sugiere que el mantenimiento sea igualmente transferido al concesionario, lo que implicaría una destinación anual de recursos económicos para su monitoreo y mantenimiento.

De igual forma, se recomienda que cuando se realice la correspondiente estimación de área equivalente a compensar, se incluya el área impactada por el efecto borde ocasionado por la fragmentación. Para esto, el ANLA o quien haga sus veces, podrá emplear los estudios científicos realizados y publicados para ecosistemas similares y así determinar cuál sería el área total para incluir a la hora de estimar un área equivalente más aproximada.

Para lograr lo anterior, se deben tener en cuenta las experiencias previas en compensaciones y los sistemas de gestión ambiental, como son las compensaciones del sector de la minería, cuya aplicación es sencilla: únicamente, la relacionada con cantidad de material de biomasa perdido y que según su importancia en 
el ecosistema es de mínimo 3 a 1 (tres especímenes por cada espécimen deteriorado); sin embargo, al desarrollar la investigación por medio de entrevistas a las agencias de minería y la ANLA, se encuentra que en algunos casos no se hace con los mismos especímenes y se termina, como ocurre con las compensaciones de la infraestructura constructiva, pagando la correspondiente multa económica (pecuniaria) o social (capacitaciones o construcciones de parques), lo cual no contribuye a la preservación de la biodiversidad y menos de los ecosistemas.

Por último, con respecto a las exigencias del programa 4 de biodiversidad y servicios ecosistémicos, de la Guía general de gestión y manejo ambiental, se debe tener en cuenta que al desarrollar las inspecciones del caso, las compañías muestran la documentación necesaria para asegurar el cumplimiento de la misma; no obstante, en la observación del entorno se determina que el cumplimiento no es tan alto y real, por lo cual es apremiante llegar a una sensibilización de la importancia de cumplir con lo establecido en el Manual de compensaciones, ya que lo anterior genera que se agoten los ecosistemas como sucede en el Cauca y en La Guajira, y en la construcción con el poco respeto por los ecosistemas como ocurre en la actualidad con los cerros orientales en Bogotá con protección legal con la Resolución 0463 de 2005 y la reserva de Van der Hammen con regulación tipo Resolución 1640 del 24 de noviembre de 2004.

\section{Conclusiones}

El Manual de compensación es una buena aproximación para la conservación de la biodiversidad; sin embargo, no son claros los resultados esperados sobre la no pérdida neta de la biodiversidad, cuando se desconocen realmente los impactos ocasionados por proyectos que requieren licencia ambiental en términos de pérdida de biodiversidad y las evaluaciones de equivalencia ecosistémica se hacen con base en coberturas y biomas, desconociendo el estado real de los ecosistemas por impactar.

De manera particular para proyectos lineales, el Manual de compensación genera varias dificultades frente a su aplicación, ya que los impactos de este tipo de proyectos se ven en diferentes ecosistemas, en pequeños fragmentos, y no contempla el impacto adicional ocasionado por la fragmentación. Sumado a esto, el tiempo corto de la duración de estos proyectos, no permite realizar el suficiente monitoreo y seguimiento a las implementaciones de compensación.

La fragmentación de los ecosistemas por la infraestructura vial, no se tiene contemplada con claridad en el otorgamiento de las licencias ambientales que se entregan a las concesiones viales; por lo tanto, se puede compensar este impacto por medio del diseño de instrumentos que mitiguen el efecto de los atropellamientos y la reducción de la capacidad reproductiva de las especies, con instrumentos de gestión que se pueden programar, planear e integrar al proyecto general, de tal forma, que este sea parte global de la concesión y quede integrado en los costos del mismo, para que, con ello, la interventoría de la concesión monitoree constantemente.

Las compensaciones in situ, para el caso de las compensaciones de proyectos viales, serían una mejor alternativa, teniendo en cuenta los impactos no contemplados por la fragmentación de los ecosistemas naturales y sería ideal si se puede transferir la responsabilidad del mantenimiento de dicha infraestructura, al concesionario. Esto no está muy lejano de lo propuesto por [23], en los bancos de hábitat.

Los bancos de hábitat serían una buena alternativa para las empresas con el fin de que organizaciones privadas puedan restaurar, crear o conservar hábitats y así vender los servicios ambientales (en forma de créditos de especies, 
hábitats o ecosistemas) a un generador de impactos ambientales que requiera cumplir con una obligación legal de compensación. Una vez comprados los créditos, el generador de impactos ambientales utilizará esos créditos como medida de compensación para cumplir con su obligación legal [24].

Para que el sistema de bancos de hábitat opere de manera transparente, eficiente y efectiva, es necesario establecer algunos acuerdos mínimos para su operación. Lo anterior es clave, pues así se definen unas bases sólidas para darle suficiente claridad y, a su vez, permite que el sistema madure con el tiempo. Los acuerdos mínimos incluyen:

- La definición de la cadena de valor-roles y responsabilidades de actores.

- Definición de reglas de juego técnicas, legales $\mathrm{y}$ financieras.

- Voluntad política.

\section{REFERENCIAS}

[1] Congreso de la República de Colombia. (1994, May 14). Ley 165 de 1994 de Convenio de las Naciones Unidas sobre Diversidad Biologica. [Online]. Recuperado: http://www.minambiente.gov.co/documentos/ ley_0165_091194.pdf

[2] Businness and biodiversity offsets Programme (BBOP). "Biodiversity Offset Design Handbook". Washington, D.C.: BBOP, 2009.

[3] MADS. "Política Nacional para la Gestión Integral de la Biodiversidad y sus Servicios Ecosistémicos". Bogotá: Imprenta Nacional, 2012.

[4] Millenium Ecosystem Assessment (MEA). "Ecosystems and Human Well-being. 4 volumes". EE.UU.: Island Press, 2005.

[5] Ministerio de Ambiente $Y$ Desarrollo Sostenible. (2014, Ene 01). Subportal Biodiversidad y Servicios Ecosistémicos. [Online]. Recuperado: www.minambiente.gov.co//contenido/contenido. aspx?catID $=1097 \&$ conID $=5551$

[6] Ideam - Instituto de Hidrología, Meteorología y Estudios Ambientales, IGAC - Instituto Geográfico
Agustín Codazzi, IAvH - Instituto de Investigación de Recursos Biológicos Alexander von Humboldt, Invemar - Instituto de Investigaciones Marinas y Costeras. "Ecosistemas continentales, costeros y marinos de Colombia”. Bogotá, D.C., 2007, 276 pp + 37 hojas cartográficas.

[7] Ministerio de Ambiente y Desarrollo Sostenible. (2014, Feb 02). Política Nacional para la Gestión Integral de la Biodiversidad y los Servicios Ecosistémicos. [Online]. Recuperado:https://www.siac. gov.co/documentos/DOC_Portal/DOC_Biodiversidad/010812_PNGIBSE_2012.pdf

[8] MADS, ANLA, TNC, WWF \& Cl. "Manual para la Asignación de Compensaciones por Pérdida de Biodiversidad". Bogotá D.C.: Ministerio de Ambiente y Desarrollo Sostenible, 2012.

[9] Ministerio de Ambiente, Vivienda y Desarrollo Territorial. (2010, Ago 05). Decreto 2820. [Online]. Recuperado: http://www.anla.gov.co/documentos/Gaceta/ Decreto_2820_de_2010.pdf

[10] Andrade, G., Sandino, J. \& Aldana, J., "Biodiversidad y territorio: innovación para la gestión adaptativa frente al cambio global, insumos técnicos para el Plan Nacional para la Gestión Integral de la Biodiversidad y los Servicios Ecosistémicos". Bogotá: MAVDT; IAVH, 2011, p. 64.

[11] Maestría en Gerencia Ambiental. "Programa Curso Proyecto de Grado". Bogotá D.C.: Universidad de los Andes, 2014.

[12] Sistema de Información Ambiental de Colombia. (2011, Ene 11). SIAC. Mapa de Coberturas Nacionales. [Online]. Recuperado:https://www.siac.gov.co/ contenido/contenido.aspx?catID $=905 \&$ conID $=1450$

[13] Mavdt, M. D Plan Nacional de Restauración. Colombia: Imprenta Nacional, 2013.

[14] Rueda Castañeda, H.V. "Contribución al Análisis del Proceso de Gestión Ambiental en Proyectos de Infraestructura Vial por Concesión a través del Estudio de Caso Briceño - Tunja - Sogamoso". Bogota: Pontificia Universidad Javeriana, 2009.

[15] Gascón, C. B. "Deforestation and forest fragmentation in the amazon". The Amazon. En Lessons from Amazonia: The ecology and conservation of a fragmented, Yale: R. O. Bierregaard, 2000.

[16] Echeverry M. "Fragmentación y deforestación como indicadores del estado de los ecosistemas en el Corredor de Conservación Choco-Manabí (ColombiaEcuador)." Recursos Naturales y Ambiente, No. 58: 58-78, 2009.

[17] Echeverry, M. "Análisis de un Paisaje Fragmentado como Herramienta para la Conservacion de la Biodiversidad en Áreas de Bosque Seco y Subhumedo Tropical en el Municipio de Pereira, Risaralda Colombia". Scientia et Technica Año XII, No 30, 405 - 410, 2006.

[18] Castaño-Uribe, C. "ías, bosques y conflicto social en Colombia.. Guerra, sociedad y medio ambiente". Bogota: M. Cárdenas \& M. Rodríguez, 2004. 
[19] Andrade, G. I., \& Castro, L. G. "Degradación, pérdida y transformación de la biodiversidad continental en Colombia, invitación a una interpretación socioecológica” Bogotá: Ambiente y Desarrollo XVI (30), 53:71, 2012.

[20] Kattan, G "Fragmentación : patrones y mecanismos de extinción de especies". Cartago: Ecología y conservación de bosques neotropicales. Ed LUR, 2002.

[21] Fuentes, E. “¿Qué futuro tienen nuestros bosques? Hacia la gestión sustentable del paisaje del centro y sur de Chile". Santiago. Chile.: Ediciones Universidad Católica de Chile, 1994.

[22] Lidicker, W. Z. Jr. "Responses of mammals to habitat edges: an overview". New york: Landscape Ecology, 14: 333-343, 1999.

[23] CEPAL. "Evaluaciones del desempeño ambiental. Colombia". Naciones Unidas, 2014.

[24] Sarmiento, M. "Los Bancos de Hábitat Como Herramienta de Compensacion en Colombia." Bogota: Fundepúbliico, 2014. 